\title{
Bone marrow-derived monocyte lineage cells recruited by MIP-1 $\beta$ promote physiological revascularization in mouse model of oxygen-induced retinopathy
}

\author{
Keijiro Ishikawa ${ }^{1}$, Shigeo Yoshida ${ }^{1}$, Shintaro Nakao ${ }^{1}$, Yukio Sassa ${ }^{1}$, Ryo Asato ${ }^{1}$, Riichiro Kohno ${ }^{1}$, Mitsuru Arima ${ }^{1}$, \\ Takeshi Kita', Ayako Yoshida', Kenoki Ohuchida² and Tatsuro Ishibashi ${ }^{1}$
}

Recent clinical observations have indicated that vascular endothelial growth factor (VEGF) is a key factor that stimulates the development of preretinal pathological neovascularization (NV). However, it has not been established how intraretinal physiological revascularization of hypoxic avascular areas is regulated. Our earlier study on the gene expression profile of hypoxic retinas in a mouse model of oxygen-induced retinopathy (OIR) showed that macrophage inflammatory protein-1 $\beta$ (MIP-1 $\beta$ ) was the most upregulated protein. The purpose of this study was to investigate the role played by MIP-1 $\beta$ in recruiting bone marrow-derived monocyte lineage cells (BM-MLCs) in a mouse model of OIR. Our results showed that MIP-1 $\beta$ was upregulated, and its receptor, CCR5, was expressed in BM-MLCs in the hypoxic inner retina. Neutralizing Ab against MIP-1 $\beta$ reduced the infiltration of BM-MLCs into the OIR retinas and increased the avascular area and preretinal neovascular tufts. A very strong significant correlation was found between the area of the preretinal neovascular tufts and the avascular area, regardless of the extent of BM-MLC infiltration into the OIR retinas. Additional treatment with VEGF-A-neutralizing Ab showed that the MIP-1 $\beta$-regulated pathological NV strongly depended on VEGF-A, which was probably secreted by the hypoxic avascular retinas. These results indicate that MIP-1 $\beta$ is involved in the recruitment of BM-MLCs, which have a significant role in the physiological revascularization of hypoxic avascular retinas. Overall, these findings indicate that the MIP-1 $\beta$ induction of BM-MLCs might possibly be used to promote intraretinal revascularization and thus prevent the abnormal NV in ischemic vision-threatening retinal diseases.

Laboratory Investigation (2012) 92, 91-101; doi:10.1038/labinvest.2011.141; published online 12 September 2011

KEYWORDS: angiogenesis; chemokines; inflammation; ischemic retinopathy; macrophage/microglia

Tissue hypoxia is believed to be the common mechanism that initiates a series of events leading to compensatory angiogenesis. Hypoxia-induced retinal neovascularization (NV) is the main contributor to proliferative vascular diseases, such as diabetic retinopathy, retinopathy of prematurity, and retinal vein occlusion. These diseases can lead to irreversible damage to visual function.

To determine the factors that are activated during retinal hypoxia, we performed a gene expression profile of hypoxic retinas obtained from a mouse model of oxygen-induced retinopathy (OIR) using gene microarray analyses. The results identified the functional set of genes that are related to the post-ischemic inflammation, neural and vascular development, and subsequent pathological NV. The most upre- gulated gene among the differentially expressed genes in hypoxic retinas was the macrophage inflammatory protein$1 \beta$ (MIP-1 $\beta)$ and not vascular endothelial growth factor A (VEGF-A) and other chemokines. ${ }^{1}$

MIP-1 $\beta$, also known as chemokine CC motif ligand 4 , is a member of the CC chemokine family that are characterized by their ability to direct migration of leukocytes into inflamed tissues. ${ }^{2}$ MIP-1 $\beta$ was first isolated from the culture medium of LPS-activated macrophages, ${ }^{3}$ and it recruited macrophages/microglia to the sites of injury in patients with arthritis, ${ }^{4}$ sepsis, ${ }^{5}$ and systemic sclerosis. ${ }^{6}$

The upregulation of MIP- $1 \beta$ in the ischemic retina has been shown to occur in an ischemia-reperfusion injury model, $^{7}$ and its receptor, CCR5, is involved in the

\footnotetext{
${ }^{1}$ Department of Ophthalmology, Kyushu University Graduate School of Medical Sciences, Fukuoka, Japan and ${ }^{2}$ Department of Surgery and Oncology, Kyushu University Graduate School of Medical Sciences, Fukuoka, Japan

Correspondence: Dr S Yoshida, MD, PhD, Department of Ophthalmology, Kyushu University Graduate School of Medical Sciences, Higashi-ku, Fukuoka 812-8582, 
development of corneal NV. ${ }^{8}$ However, it is not known what role the MIP-1 $\beta /$ CCR5 system has in the hypoxia-induced retinal NV.

There are two types of hypoxia-induced retinal NV; one is pathological NV with a sprouting of abnormal vessels from the retina into the vitreous, and the other is physiological revascularization of avascular areas with functional intraretinal vasculature. Recent experimental and clinical studies have shown that VEGF-A is a key factor in the development of pathological NV, and VEGF-A inhibitory drugs can inhibit the pathological NV, including those associated with retinal diseases. ${ }^{9,10}$ However, the clinical use of VEGF-A inhibitors showed that these drugs can also enhance tissue hypoxia that would then damage the tissue. ${ }^{11,12}$

Little information is available on how physiological revascularization of hypoxic avascular retinas is regulated. Bone marrow-derived monocyte lineage cells (BM-MLCs) are important angiogenic effector cells that produce a number of proangiogenic factors. ${ }^{13}$ The role played by proangiogenic BM-MLCs in hypoxia-induced retinal NV has been discussed mainly in the process of pathological $\mathrm{NV}^{14}$ It has been suggested that the SDF1/CXCR4 system might be involved in the recruitment of these cells into hypoxic retinas. ${ }^{15}$

The ability of the retina to respond to hypoxia by physiological revascularization suggests that this property of the retina might be used in the treatment of ischemic retinopathy. In this process, BM-MLCs would facilitate the physiological revascularization in the hypoxic areas. ${ }^{16}$ However, little is known on the mechanism that recruits these cells into hypoxic retinas.

Thus, the purpose of this study was to investigate the role played by the MIP-1//CCR5 system in hypoxia-induced retinal NV. We shall show that BM-MLCs promoted intraretinal revascularization in hypoxic avascular retina. The possible biological role played by BM-MLCs in the formation of intraretinal revascularization is discussed.

\section{MATERIALS AND METHODS Mouse Model of OIR}

All experimental procedures on the animals were performed according to the Association for Research in Vision and Ophthalmology Statement for the Use of Animals in Ophthalmic and Vision Research. OIR was induced in C57BL/6J mice as described in detail by Smith et al. ${ }^{17}$ Briefly, litters of postnatal day 7 (P7) C57BL/6J pups along with their mothers were placed in a $75 \pm 2 \%$ oxygen atmosphere (hyperoxia) for 5 days and then returned to room air at age P12. After the pups were returned to room air and the avascular areas of retina became hypoxic, intraretinal physiological revascularization of the avascular areas and preretinal pathological NV developed simultaneously. Pathological NV reached its maximum at P17. Mice were killed by cervical dislocation, and the eyes were enucleated.

\section{RNA Isolation and Real-Time Quantitative Reverse Transcription-Polymerase Chain Reaction}

Whole retinas free of vitreous were isolated from normal P12 and hyperoxic mice before returning them to room air. Other mice were kept in room air for 3, 6, $12 \mathrm{~h}$, and 1, 2, 3, and 5 days (relative hypoxia) before the retinas were isolated. Two retinas from one mouse were pooled, and three experiments for each condition (biological replicates) were examined. RNA isolation and real-time quantitative reverse transcription-polymerase chain reaction (qRT-PCR) were performed as described. ${ }^{1}$ The primers were as follows: for Mip- $1 \beta$, 5'-CCATGAAGCTCTGCGTGTCTG-3' ${ }^{\prime}$ and $5^{\prime}$-GGCTTGGAG CAAAGACTGCTG-3'; for F4/80, 5'-CGCTGCTGGTTGA ATACAGAGA- $3^{\prime}$ and $5^{\prime}$-CGGTTGAGCAGACAGTGAATG A-3'; for Vegfa, 5'-GGAGAGCAGAAGTCCCATGA-3' and $5^{\prime}$-ACTCCAGGGCTTCATCGTTA- ${ }^{\prime}$; and for GAPDH, $5^{\prime}$-GT GAAGGTCGGTGTGAACGG- $3^{\prime}$ and $5^{\prime}$-TCTCCATGGTGGT GAAGACA- $3^{\prime}$.

\section{Enzyme-Linked Immunosorbent Assay}

Whole retinas free of vitreous were isolated from normal P12 mice, hyperoxic mice P12 mice, and from mice after $6,12 \mathrm{~h}$, and $1,2,3$, and 5 days of relative hypoxia. Four retinas from two mice raised under the same conditions were pooled, and three experiments for each condition (biological replicates) were examined. Protein was isolated as described. ${ }^{1}$ An ELISA kit (R\&D Systems, Minneapolis, MN) was used to measure the concentrations of MIP- $1 \beta$ according to the manufacturers' instructions. The minimum concentration of MIP$1 \beta$ that could be detected was $6.0 \mathrm{pg} / \mathrm{ml}$. The concentration of total protein was determined with a commercial assay kit (Bio-Rad protein assay reagent kit; Bio-Rad Laboratories, Hercules, CA).

\section{Microdissection-Based Quantitative Analysis of mRNA}

The retinal layers were dissected by laser capture microdissection (LCM) to analyze the MIP- $1 \beta$ expression in the retinal ganglion cell layer (GCL), inner nuclear layer (INL), and outer nuclear layer (ONL). Frozen eyes of P12 and $\mathrm{P} 12+12-\mathrm{h}$ control mice, P12 hyperoxia-exposed mice, and mice at $12 \mathrm{~h}$ after relative hypoxia were cut into $8-\mu \mathrm{m}$ thick sections. The GCL, INL, and ONL in the retinas at each time were isolated selectively using LCM and a pressure catapulting system (LMPC; Palm Microlaser Technologies AG, Bernried, Germany) in accordance with the manufacturer's protocols. After microdissection, the total RNA was extracted from the selected cells as described, ${ }^{18}$ and real-time qRT-PCR for MIP- $1 \beta$ was performed on the RNA. The results were normalized to the level of GAPDH expression.

\section{Immunohistochemistry of Retina}

Immunohistochemistry was performed as described. ${ }^{19}$ The eyes of hypoxic P14 mice and normoxic P14 mice were enucleated and fixed in $4 \%$ paraformaldehyde (PFA) and then embedded in paraffin. Sections $(3 \mu \mathrm{m})$ were rehydrated 
and incubated for $1 \mathrm{~h}$ at room temperature with anti-MIP- $1 \beta$ antibody (1:500 dilution; R\&D Systems, Minneapolis, MN) or antibody against VEGF (1:100 dilution; Santa Cruz Biochemicals, Santa Cruz, CA) with $1 \%$ skim milk. The bound $\mathrm{Ab}$ was made visible by a conventional avidin-biotin peroxidase protocol with 3-amino-9-ethylcarbazole as the substrate. For negative controls, mouse nonimmune IgG was used as the primary Ab.

\section{Immunofluorescent Staining}

For FITC-dextran angiography, animals were anesthetized by an intraperitoneal injection of sodium pentobarbital, and approximately $500 \mu \mathrm{l}$ of FITC-dextran $(50 \mathrm{mg} / \mathrm{ml}$; SigmaAldrich, St Louis, MO) was injected into the left ventricle. The dextran was allowed to circulate for approximately $2 \mathrm{~min}$ before the animals were euthanatized and the eyes enucleated. Immunofluorescent staining was done for the following: F4/80, a marker for monocyte lineage cells; CCR5, the sole receptor for MIP-1 $\beta$ ligand; and CD45, a marker commonly used to identify BM-derived cells ${ }^{20}$ on P17 OIR retinas. After the eyes were enucleated, the cornea, iris, and sclera were removed leaving the retina around the lens. After an initial fixation in $4 \%$ PFA for $1 \mathrm{~h}$ at $4{ }^{\circ} \mathrm{C}$, the lens was removed. The whole retinas were then fixed in the same fixative for an additional $21 \mathrm{~h}$ at $4{ }^{\circ} \mathrm{C}$. Fixed samples were rinsed with phosphate-buffered saline (PBS) and placed in $100 \%$ methanol at room temperature for $10 \mathrm{~min}$. The samples were blocked using 5\% skim milk in PBS for $1 \mathrm{~h}$ at room temperature, and then incubated in primary $\mathrm{Abs}$ at $4{ }^{\circ} \mathrm{C}$ for $24 \mathrm{~h}$ and then in the secondary $\mathrm{Ab}$ for $1 \mathrm{~h}$ at room temperature. Radial cuts were made in the retinas, and the retinas were flat-mounted in Crystal/Mount (Biomedia, Foster City, CA). The retinas were examined by fluorescent microscopy (BZ-9000; KEYENCE, Osaka, Japan).

The primary Abs were F4/80 (1:1000 dilution, rat IgG; eBIOSCIENCE, San Diego, CA, USA), rabbit/anti-mouse CCR5 Ab (1:1000 dilution, rabbit IgG; Abcam, Cambridge, MA, USA), and anti-mouse CD45-cy5 Ab (1:1000 dilution; Abcam). The secondary Abs were AlexaFluor546 (1:800 dilution, chicken anti-rat IgG; Molecular Probes), AlexaFluor647 (1:800 dilution, goat anti-rabbit IgG; Molecular Probes), AlexaFluor488 (1:800 dilution, chicken anti-rabbit IgG; Molecular Probes), and AlexaFluor546 (1:800 dilution, goat anti-rat IgG; Molecular Probes).

\section{Intravitreal Injections}

Mice were deeply anesthetized by an intraperitoneal injection of sodium pentobarbital. The closed lids were separated, and the eyes were proptosed. Intravitreal injections were performed on $\mathrm{P} 12$ and $\mathrm{P} 14$ pups by injecting $1.0 \mu \mathrm{l}$ of the agents into the eyes with a 32 -gauge needle on a Hamilton syringe. The needle was inserted $200 \mu \mathrm{m}$ posterior to the limbus. For combined injections of anti-MIP- $1 \beta$ and anti-VEGF ${ }_{164} \mathrm{Ab}$, anti-MIP- $1 \beta \mathrm{Ab}$ was injected into P12 pups and $1.0 \mu \mathrm{l}$ of premixed Abs (50 ng/0.5 $\mu$ l each) was injected into P14 pups.
The agents were neutralizing Ab against MIP- $1 \beta(100 \mathrm{ng} / \mu \mathrm{l}$; $\mathrm{R} \& \mathrm{D}$ Systems) and neutralizing Ab against $\mathrm{VEGF}_{164}$ (100 ng/ $\mu \mathrm{l}$; R\&D Systems). Nonimmune IgG was used as controls.

\section{Quantification of F4/80 mRNA Expression and F4/80 Positive Cells}

Retinas were dissected from P17 OIR pups after the injections of the different agents, and RNA analysis and immunofluorescent staining were performed as described. To quantify the relative mRNA expression of F4/80 normalized to GAPDH, real-time qRT-PCR was performed on MIP-1 $\beta$ antibody-treated retinas $(n=6)$ and nonimmune IgG-treated controls $(n=6)$. To quantify the F4/80-positive cells in P17 OIR flat-mounted retinas, photographs were taken at $\times 4$ magnification (4 images/retina), and the F4/80-positive cells were counted in the digital images in a masked manner (MIP-1 $\beta \mathrm{Ab}, n=8$; control, $n=8$ ).

\section{Quantification of Avascular Areas and Neovascular Tuft Areas}

Quantification of the avascular areas and neovascular tuft areas in P17 OIR retinas was performed according to a described protocol. ${ }^{21}$ After the injections of the different agents into P17 OIR eyes, the retinas were flat-mounted and stained with fluorescein-labeled isolectin B4 (1:150 dilution; Vector Laboratories, Burlingame, CA). The retinas were photographed under a fluorescent microscope (BZ-9000; KEYENCE) and merged to obtain whole-mount retinal images using image-joint software BZ-Analyzer (KEYENCE).

\section{Statistical Analyses}

All results are expressed as the means \pm standard error of the means. One-way ANOVA followed by Bonferroni $t$ tests was used to compare the MIP- $1 \beta$ mRNA expression level with that of the control retinas at each time point. Student's $t$-tests were used to compare the control OIR retinas with those given MIP- $1 \beta \mathrm{Ab}$. Dunnett's multiple-comparison tests were used to compare the areas of avascular retinas and neovascular tufts among groups. Pearson product-moment correlation coefficients were calculated to determine the correlations between the pathological and physiological NV. A value of $P<0.01$ was considered statistically significant. Statistical analyses were performed using JMP version 7.0.1 (SAS Institute, Cary, NC).

\section{RESULTS}

\section{Induction of MIP-1 $\beta$ in OIR Retinas}

We have shown that the expression of the MIP-1 $\beta$ gene was the most upregulated compound in the hypoxic retinas of mouse OIR mice. ${ }^{1}$ To confirm the upregulation of the mRNA and protein levels of MIP- $1 \beta$ in OIR mice, we performed real-time qRT-PCR and enzyme-linked immunosorbent assay (ELISA), respectively, on retinal extracts. Real-time qRTPCR showed that the mRNA of MIP- $1 \beta$ was slightly increased in the retinas of mice 5 days after hyperoxia (P12), and the 
level increased and peaked at 1 day after hypoxia. The level of the mRNA of MIP-1 $\beta$ then gradually decreased (Figure 1a). The MIP-1 $\beta$ protein was undetectable until $6 \mathrm{~h}$ after the hypoxic exposure, but markedly increased and peaked on day 2 , and then gradually decreased. The protein levels in the P12 control retinas and after 5 days of hyperoxia were below the detectable level (Figure 1b).

\section{Localization of MIP-1 $\beta$ in OIR Retinas}

To determine the location of MIP- $1 \beta$ in the hypoxic retina, we performed real-time qRT-PCR with extracted mRNA from the LCM in the GCL, INL, and ONL at each time point. The mRNA level of MIP- $1 \beta$ was undetectable in each layer of $\mathrm{P} 12, \mathrm{P} 12+12 \mathrm{~h}$ control retinas, and from $\mathrm{P} 12$ hyperoxic retinas. At $12 \mathrm{~h}$ after relative hypoxia, the mRNA of MIP- $1 \beta$ was detected in OIR retinas, and the gene expression was prominent in the GCL and relatively weak in the ONL (Figure 2). These findings indicated that the expression of the mRNA of MIP- $1 \beta$ was upregulated in the hypoxic retina and increased in the inner layer in a hypoxic gradient.

To confirm the sites of the MIP- $1 \beta$ protein in the hypoxic retina, immunohistochemistry was performed on hypoxic
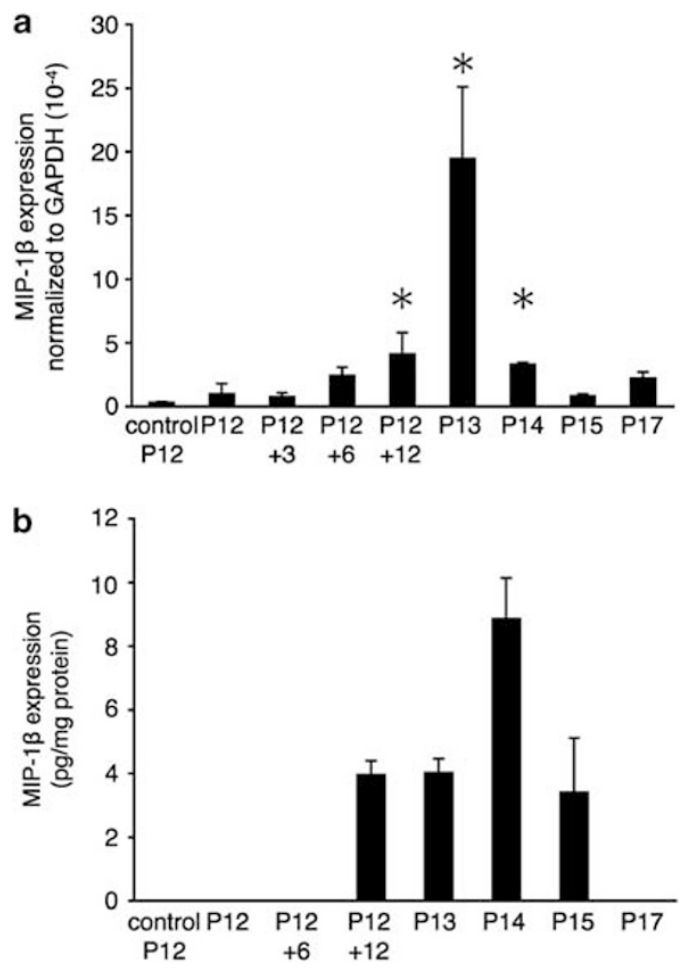

Figure 1 Kinetics of the changes in the mRNA (a) and protein levels (b) of MIP-1 $\beta$ in oxygen-induced retinopathy (OIR) retinas. The mRNA of MIP-1 $\beta$ is slightly but not significantly increased in the retinas of mice 5 days after hyperoxia (P12). The level then increases significantly, peaks at 1 day after the hypoxia and then gradually decreases (a). The protein level of MIP- $1 \beta$ is undetectable until $6 \mathrm{~h}$ after hypoxia but is then markedly increased and peaks at day 2 , and then gradually decreased $(\mathbf{b})$. ${ }^{\star} P<0.01$ compared with normal subjects.

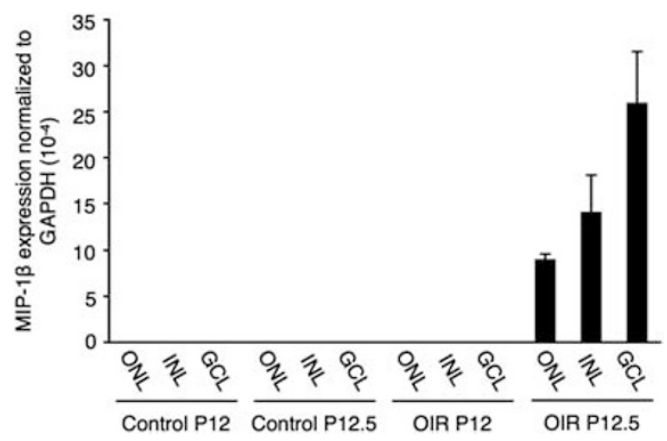

Figure 2 Expression levels of MIP-1 $\beta$ mRNAs in the retinal ganglion cell layer (GCL), inner nuclear layer (INL), and outer nuclear layer (ONL) of P12 and $\mathrm{P} 12+12$-h control retinas, OIR P12 hyperoxic retinas, and OIR retinas at $12 \mathrm{~h}$ after hypoxia. The relative level of mRNA expression is quantified and normalized to the levels of GAPDH mRNA expression. The MIP-1 $\beta$ mRNA level was undetectable in all layers of both $\mathrm{P} 12, \mathrm{P} 12+12$-h control retinas and $\mathrm{P} 12$ hyperoxic retinas. At $12 \mathrm{~h}$ after relative hypoxia, the mRNA of MIP$1 \beta$ was detected in the OIR retinas, and the distribution of the gene expression was prominent in the GCL and relatively low in the ONL.

and normoxic P14 retinas. Signals were observed in the hypoxic inner retina and most strongly in the GCL. The cells located in the INL showed relatively weak signals (Figure 3a). In contrast, only very weak signals were observed in the GCL of normal retinas (Figure 3b). As a positive control, immunoreactivity for VEGF-A, the representative hypoxiainduced protein in retina, was performed. Consistent with earlier studies, ${ }^{22}$ VEGF-A signals were observed in all layers of both control and hypoxic retinas, but especially strongly in the hypoxic inner retina (Figure $3 \mathrm{c}$ and $\mathrm{d}$ ).

\section{CCR5 Expression in BM-MLC Infiltrating OIR Retinas}

To determine the distribution and morphology of monocyte lineage cells that were recruited by MIP- $1 \beta$ into the OIR retinas, immunofluorescent staining for $\mathrm{F} 4 / 80$ combined with FITC-dextran angiography was performed. In the whole mounts of P17 OIR retinas, amoeboid F4/80 + cells possessing thin filopodia-like processes, called 'activated microglias, ${ }^{23}$ and round-shaped $\mathrm{F} 4 / 80+$ cells, monocyte lineage cells, were detected at the border between vascular and avascular areas (Figure 4a). A few F4/80 + cells were also seen in the avascular area.

To confirm the presence of MIP- $1 \beta$-inducible BM-derived $\mathrm{F} 4 / 80+$ cells, immunofluorescent staining for F4/80, CCR5, and CD45 was performed. The round-shaped F4/80 + cells distinctly coexpressed CCR5 (Figure $4 \mathrm{~b}-\mathrm{d}$ ). In contrast, the signal for CCR5 was negative in most of the amoeboid F4/ $80+$ cells, but seen weakly in suborbicular cells with short processes that appeared to be differentiating from monocytes to amoeboid microglias (arrowhead, Figure 4b-d). Expression of CD45 was seen in the F4/80+ round-shaped or suborbicular cells with short processes (Figure 4e-g). Aggregates of round-shaped CCR5 + cells (Figure 4h) and CD45 + cells (Figure 4i) were seen in P17 OIR retinas, and 

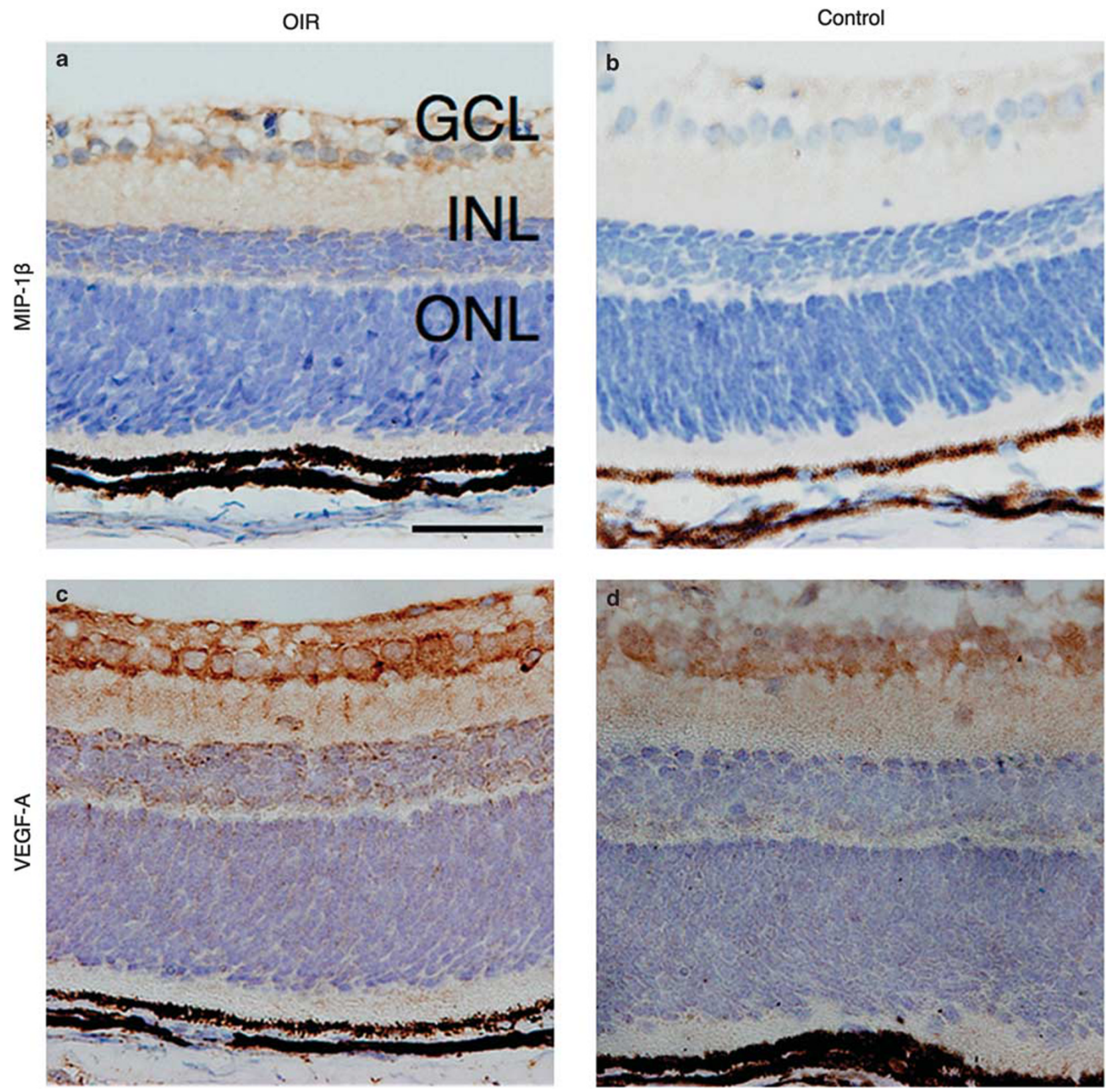

Figure 3 Immunohistochemical staining for MIP-1 $\beta$ and VEGF-A in the hypoxic OIR retinas. Signals are observed in the hypoxic inner retina and most strongly in the GCL, and the cells located in the INL showed relatively weak signals (a). In contrast, only a very weak signal is observed in the GCL of the normal retina (b). The signal of VEGF-A is observed in all layers of both control and hypoxic retinas (c, d). $\mathbf{a}$ and $\mathbf{c}$, retina 2 days after hypoxia (P14); $\mathbf{b}$ and $\mathbf{d}$, control normal retina (P14). Original bar $=50 \mu \mathrm{m}$.

the merged images showed that most of the cells expressed both (Figure $4 \mathrm{j}$ ). These data suggest that CCR5, the sole receptor for MIP-1 $\beta$ ligand, was expressed in the BM-MLC infiltrating the P17 OIR retinas.

\section{Blockade of MIP-1 $\beta$ Inhibits F4/80 + Cells Infiltration into OIR Retinas}

To determine whether MIP- $1 \beta$ was involved in the recruitment of monocyte lineage cells into OIR retinas, we ex- amined whether neutralization of MIP-1 $\beta$ can reduce the number of F4/80 + round-shaped or suborbicular cells and the mRNA expression of F4/80 in the P17 OIR retinas. In the P17 OIR flat-mounted retinas exposed to neutralizing Abs against MIP- $1 \beta$, the F4/80 + round-shaped or suborbicular cells were decreased significantly more than in the retinas injected with control nonimmune $\operatorname{IgG}(26.8 \%$ decrease, $n=8, P<0.05$; Figure 5a-e). In agreement, real-time qRTPCR also showed a significant reduction in F4/80 mRNA 

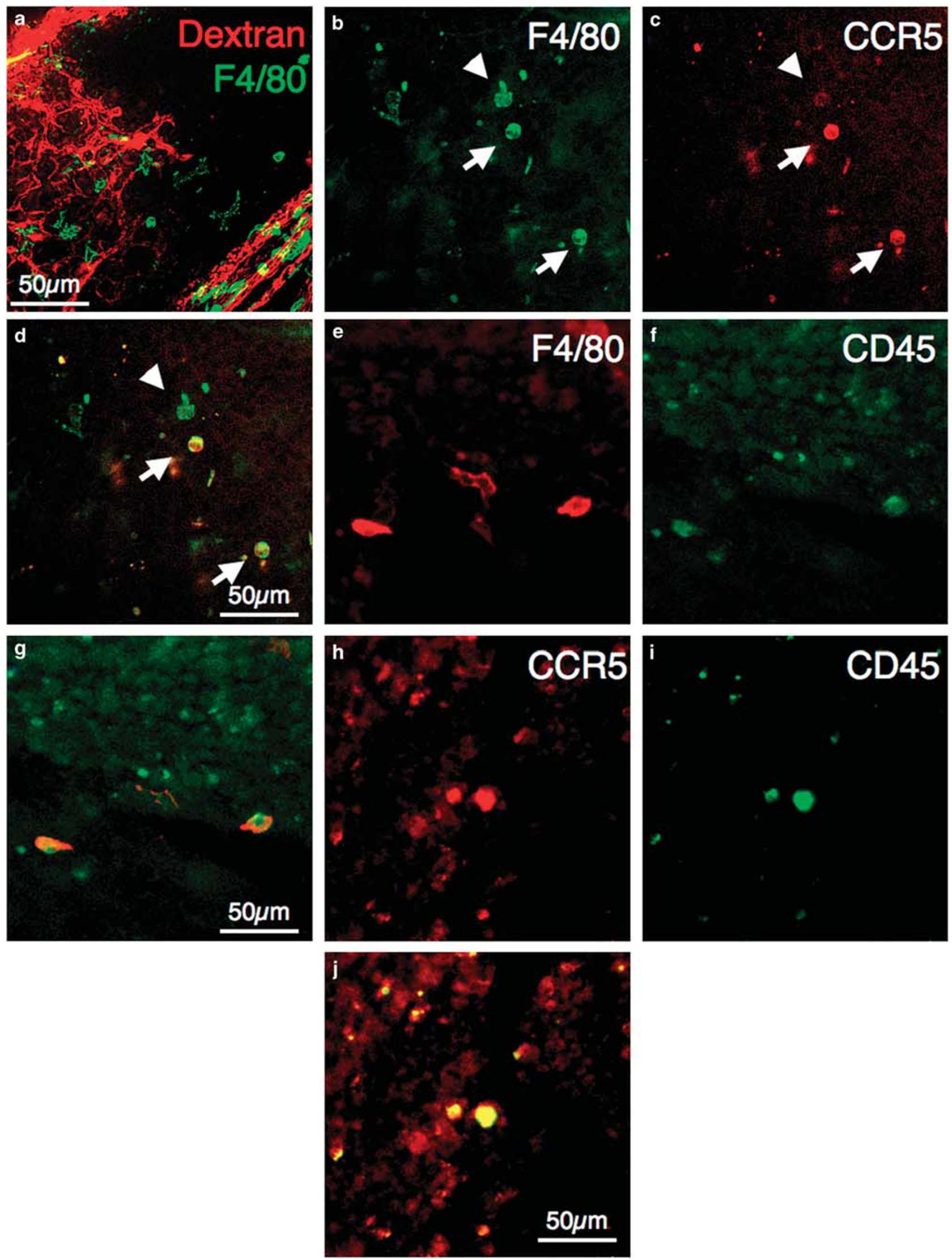

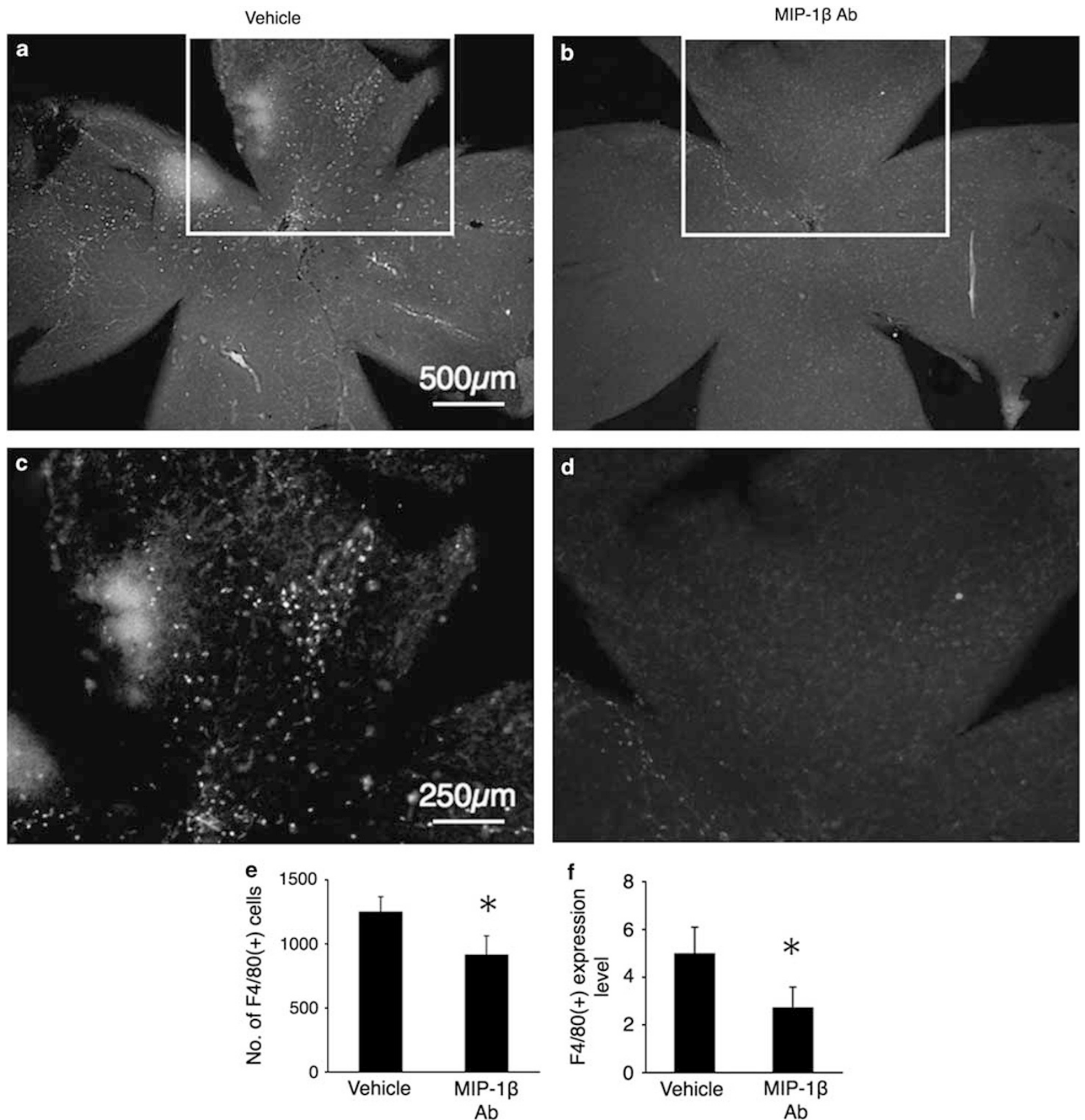

Figure 5 Results of quantification of F4/80 + cells and F4/80 mRNA level in P17 OIR retinas. MIP-1 $\beta$-neutralizing Ab was injected at P12 and P14. At P17, the whole retinas were immunofluorescently stained with $\mathrm{F} 4 / 80$. The low-power views $(\mathbf{a}, \mathbf{b})$ provide perspective, and the square box regions are shown in the higher magnification views (c, d). Compared with retinas from mice treated with vehicle $(\mathbf{a}, \mathbf{c})$, retinas from mice treated with the $A b$ had fewer $\mathrm{F} 4 / 80+$ cells $(\mathbf{b}$, d). Image analysis confirmed that there were significantly fewer $\mathrm{F} 4 / 80+$ cells $(\mathbf{e}, n=8)$ in retinas from eyes treated with the Ab than fellow eyes treated with vehicle. Quantitative real-time PCR for F4/80 mRNA expression normalized to GAPDH showed significantly fewer in P17 OIR retinas treated with the $\mathrm{Ab}$ than fellow eyes treated with vehicle $(\mathbf{f}, n=6) .{ }^{\star} P<0.01$.

Figure 4 CCR5 expression in BM-MLCs infiltrating the P17 OIR retinas. Amoeboid microglias and round-shaped monocyte lineage cells are located at the border between vascular and avascular areas (a). The round-shaped F4/80 + cells (arrows) and the suborbicular F4/80 + cells with short processes (arrow head) coexpress CCR5 (b-d). The round-shaped F4/80 + cells or suborbicular F4/80 + cells with short processes coexpress CD45 (e-g).

The round-shaped CCR5 + cells coexpress CD45 (h-j). 
expression in the retinas exposed to Abs compared with the control P17 OIR retinas (45.7\% decrease, $n=6, P<0.05$; Figure $5 \mathrm{f}$ ). These results indicated that MIP- $1 \beta$ has a role in the infiltration of $\mathrm{F} 4 / 80+$ cells into OIR retinas.

\section{Blockade of MIP-1 $\beta$ Suppresses Physiological Revascularization and Promotes Pathological NV in OIR Retinas}

To examine the role played by MIP- $1 \beta$ in hypoxia-induced retinal NV, we tested the effects of neutralizing Abs against MIP- $1 \beta$ in the physiological revascularization and pathological NV in the OIR model. We measured the avascular areas representing physiological revascularization and the areas of neovascular tufts representing pathological NV in P17 OIR flat-mounted retinas given Abs against MIP- $1 \beta$. To compensate for the difference in retinal growth among mice, we divided the number of pixels in the areas by the number of pixels in the total retinal area according to the protocol of Connor. ${ }^{21}$

The relative sizes of both the avascular areas and the areas of neovascular tufts were significantly increased. These findings indicated that the physiological revascularization was suppressed and pathological NV was enhanced in the retinas injected with the Abs compared with the control OIR retinas (34.5 and $21.4 \%$ increase, respectively, $n=8$; Figure $6 a, b, d, e, g$, and h).

\section{Area of Neovascular Tufts Significantly Correlated with Area of Avascular Retina, Regardless of Extent of BM-MLC Infiltration into OIR Retinas}

In P17 OIR retinas exposed to neutralizing Abs against MIP$1 \beta$, the size of both the avascular areas and neovascular tufts significantly increased compared with the control OIR retinas. We then determined the correlation between the sizes of the avascular areas to that of the neovascular tufts to determine the contribution of hypoxic avascular retinas to the formation of pathological NV. A very strong correlation was found both in the control OIR retinas $(r=0.953, n=8$, $P<0.01)$ and the retinas injected with the neutralizing Abs against MIP-1 $\beta$ ( $r=0.944, n=8, P<0.01$; Figure $7 \mathrm{a}$ and $\mathrm{b})$. These results indicated that the formation of pathological NV strongly depended on the spread of the avascular area, regardless of the extent of BM-MLC infiltration induced by MIP-1 $\beta$.

\section{VEGF-A Upregulation has an Important Role in Promotion of Pathological NV in OIR Retinas}

It is well known that hypoxia-induced angiogenic factor VEGF-A has an important role in the pathogenesis of ischemic retinal neovascular diseases, such as diabetic retinopathy and branch retinal vein occlusion. ${ }^{9}$ To examine if VEGF-A could contribute to the MIP- $1 \beta$ suppression-induced pathological NV enhancement, we investigated the degree of expression of VEGF-A in the retinas after inhibiting MIP-1 $\beta$. The VEGF-A mRNA expression was significantly increased in the P17 OIR retinas exposed to the neutralizing Abs against MIP- $1 \beta$ compared with the control OIR retinas (79.3\% increase, $P<0.05, n=6$; Figure $6 \mathrm{i}$ ).

Additional intravitreal injections of anti-VEGF-A-neutralizing Abs with anti-MIP- $1 \beta$ Abs did not increase the sizes of the avascular areas (10.9\% increase, $n=8, P>0.01$; Figure $6 \mathrm{~g}$ ) but significantly decreased the sizes of neovascular tufts ( $80.4 \%$ decrease, $n=8, P<0.01$; Figure $6 \mathrm{~h}$ ) compared with the control OIR retinas. No significant correlation was found between the sizes of the avascular areas and those of neovascular tufts in the retinas treated with both Abs (Figure 7c).

These data suggest that the spread of the hypoxic avascular retina by MIP- $1 \beta$-inhibition-induced VEGF-A upregulation results in the promotion of pathological NV.

\section{DISCUSSION}

To the best of our knowledge, this is the first study that showed that the MIP-1 $\beta /$ CCR5 system was involved in the recruitment of BM-MLCs that promote the physiological revascularization of hypoxic avascular retinas. The involvement of BM-MLCs in physiological revascularization is in agreement with earlier studies that BM-derived cells contribute to the growth of blood vessels during postnatal vascular regeneration and vascular remodeling after injury. ${ }^{24,25}$

In the CNS, BM-MLCs and activated microglias can be seen at the border of cerebral infarcted areas in the ischemic brain, ${ }^{26}$ as we observed at the border between vascular and avascular retina (Figure 4a). The cells in the cerebral ischemic areas may secrete a variety of proinflammatory cytokines, ${ }^{27}$ neurotrophic factors, and angiogenic factors such as VEGF. ${ }^{28}$ This would then indicate that the BM-MLC-activated microglias may be involved in the pathogenesis of postischemic inflammation and subsequent $\mathrm{NV}$ in hypoxic retinas.

We observed that the levels of the mRNA and proteins of MIP- $1 \beta$ were significantly increased shortly after the avascular retinas were exposed to relative hypoxia and then gradually decreased (Figure 1). The kinetics of these changes is fairly consistent with the size of the central avascular area, which was at a maximum between P12 and P13 and gradually decreased due to revascularization. ${ }^{29}$ In ischemic retinopathies, the inner retina, which is supplied by retinal vessels, is hypoxic whereas the outer retina, which is supplied by the choroidal vessels, is not. ${ }^{30}$ Microdissection-based quantitative analysis and immunohistochemistry showed that the levels of the mRNAs and protein of MIP- $1 \beta$ increased in the inner retina in accordance with the hypoxic gradient (Figures 2 and $3)$. These results clearly demonstrated that MIP- $1 \beta$ is differentially induced both temporally and spatially by retinal hypoxia. Moreover, higher MIP-1 $\beta$ mRNA expression is associated with more $\mathrm{F} 4 / 80+$ cells in the region of the vitreoretinal interface of P14 OIR retinas. ${ }^{31}$ This distribution of the F4/80 + cells is reasonable because the physiological revascularization of hypoxic avascular retina starts from the superficial vascular plexus between the avascular and peripheral vascularized areas. ${ }^{32}$ 

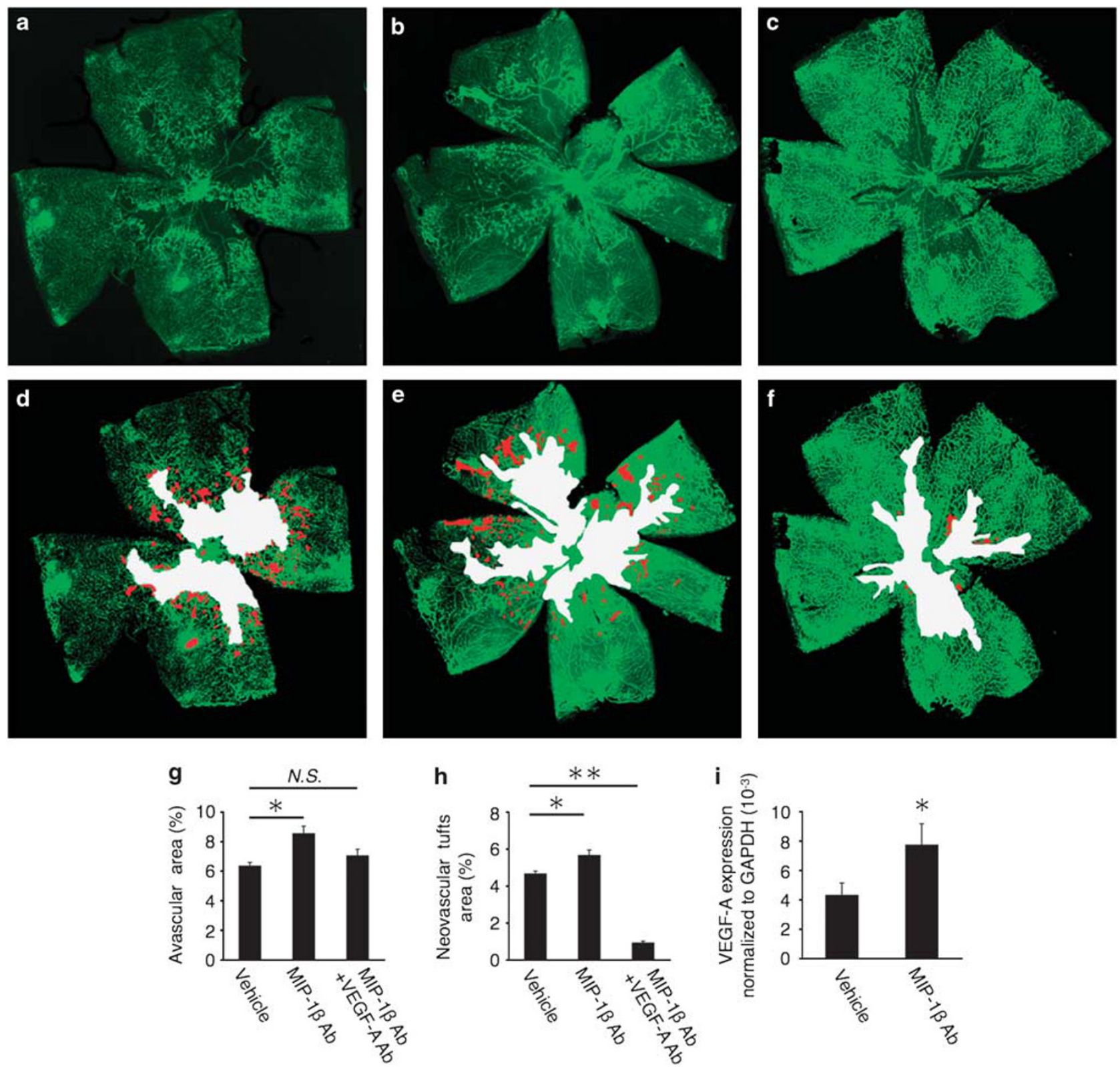

Figure 6 Results of quantification of the avascular areas and neovascular tufts in P17 OIR retinas. Representative images of a flat-mounted OIR retina $(\mathbf{a}, \mathbf{d})$, OIR retina administered with MIP-1 $\beta$-neutralizing Ab (b, e), and OIR retina coadministered with MIP-1 $\beta$ - and VEGF 164 -neutralizing Ab (c, f). The red shading represents neovascular tufts and white shading represents avascular area. In the retinas with MIP-1 $\beta$ Ab, the relative sizes of both the avascular areas and those of neovascular tufts significantly increased compared with the control OIR retinas $(\mathbf{g}, \mathbf{h}, n=8)$. In the retinas coadministered with MIP-1 $\beta$ and VEGF 164 Ab, the relative sizes of the avascular areas slightly increased (g, $n=8$, not significant) and those of neovascular tufts significantly decreased compared with the control OIR retinas $(\mathbf{h}, n=8)$. Quantitative real-time PCR for VEGF-A mRNA expression normalized to GAPDH significantly increased in P17 OIR retinas with MIP-1 $\beta$ Ab than fellow eyes treated with vehicle $(\mathbf{i}, n=6) .{ }^{\star} P<0.05,{ }^{*} P<0.01$.

The expression of CCR5, the sole receptor for MIP- $1 \beta$, appeared to be decreased with activation in the hypoxic retina signal (Figure $4 \mathrm{~b}-\mathrm{d}$ ). This finding is in line with the results of an earlier study by Bosco et al, ${ }^{33}$ who showed that hypoxia inhibits the expression of CCR5 in mouse macrophages in vitro. They proposed that the downregulation of CCR5 might represent a negative feedback mechanism that regulates leukocyte recruitment at the sites of inflammation, or an important regulatory mechanism to retain/concentrate recruited macrophages within the lesions by preventing their migration along other chemokine gradients. On the basis of the similarity of the mechanisms, the CCR5 expressing BMMLCs recruited by MIP- $1 \beta$ secreted from hypoxic retinas may possibly be retained in the region by downregulating CCR5, and subsequently activating and exerting angiogenic activities. 

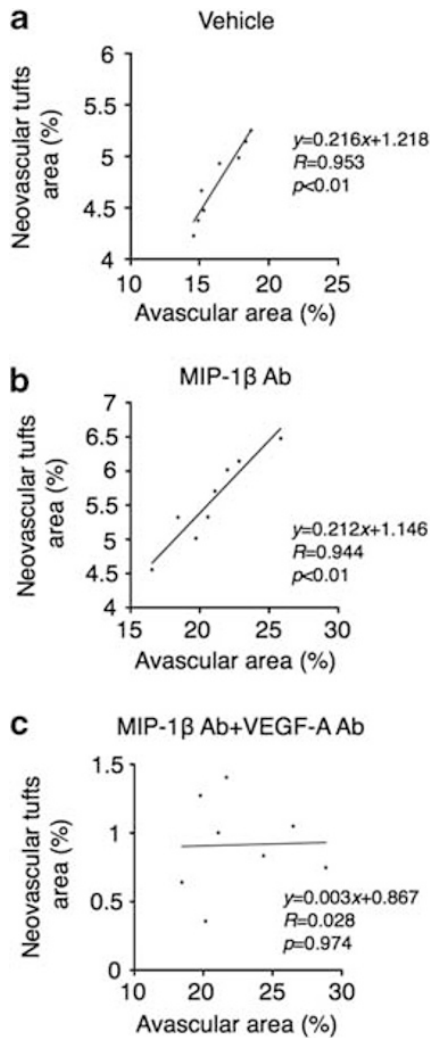

Figure 7 Correlation between the sizes of avascular areas and those of neovascular tufts in P17 OIR retinas. A very strong correlation was found both in control OIR retinas $(\mathbf{a}, n=8)$ and the retinas injected with the neutralizing Abs against MIP-1 $\beta(\mathbf{b}, n=8)$. The correlation between the retinas exposed to both MIP-1 $\beta$ - and VEGF VE4 $_{164}$-neutralizing Ab was not significant $(\mathbf{c}, n=8)$.

We have found that MIP- $1 \beta$ is involved in the recruitment of BM-MLCs into OIR retinas by showing that MIP- $1 \beta$ induces the infiltration of $\mathrm{F} 4 / 80+$ round-shaped or suborbicular cells, which were positive for CD45, a marker commonly used to identify BM-derived cells. Our finding that MIP- $1 \beta$ released from hypoxic retina recruited BMMLCs is in line with the in vitro chemotaxis assay showing that MIP- $1 \beta$ cells can induce the migration of blood monocytes. ${ }^{34}$ Other members of the chemokine family, such as MCP-1 and SDF-1/CXCR4, have been reported to regulate BM-MLC trafficking in hypoxic retinas and the subsequent pathological NV. ${ }^{15,35}$ However, the relationship between the diverse hypoxia-induced chemokines, trafficking properties of the BM-MLCs, and the variety of biological functions in hypoxic retinas are not fully understood. It is also known that VEGF contributes to the recruitment of monocytes to hypoxic tissues. ${ }^{36}$ Thus, it might be possible that the MIP- $1 \beta$ inhibition and VEGFA upregulation is a compensatory mechanism for the decrease of BM-MLC infiltration into OIR retinas.

Inhibition of BM-MLC infiltration had adverse effects on the two types of hypoxia-induced retinal NV, a suppression of physiological revascularization and promotion of pathological NV (Figure 6). These results are in accordance with a recent study demonstrating the contradictory roles of BMderived cells in physiological and pathological NV, using the OIR model with irradiation-induced leukocyte depletion. ${ }^{37}$ We proposed a mechanism that this apparent contradictory role is due to the spread of avascular area subsequent to the suppression of physiological revascularization that has much stronger effects on the pathological NV compared to that of BM-MLC infiltration (Figure 7). It is well known that hypoxic retinas alter pathological NV as shown by the strong relationship between the degree of retinal avascularity and pathological NV in the rat model of OIR. ${ }^{38}$ In addition, it has been reported that VEGF-A is an important hypoxia-induced proangiogenic factor promoting pathological $\mathrm{NV}^{22,39} \mathrm{We}$ also confirmed that VEGF-A was upregulated in the hypoxic retina, and that anti-VEGF-A-neutralizing Abs almost completely suppressed the pathological NV in the OIR retinas with broad avascular areas caused by the MIP- $1 \beta$ inhibition (Figure 6).

Taken together, our results showed that the inhibition of BM-MLC infiltration by the inhibition of MIP- $1 \beta$ suppressed the physiological revascularization of the avascular area, resulting in the expansion of the hypoxic avascular retina. The expansion of the hypoxic avascular retina increased the secretion of angiogenic VEGF and consequently promoted pathological NV. The results of pathological NV promotion induced by MIP- $1 \beta$ inhibition raise the possibility that, in the process of preretinal pathological $\mathrm{NV}$, the direct proangiogenic contribution of BM-MLCs recruited by MIP- $1 \beta$ is very small. These cells might have an antiangiogenic role, which is supported by an earlier report, showing the involvement of macrophages/microglia in pathological NV regression through apoptosis. ${ }^{35}$

The current therapeutic approaches for ocular neovascular diseases mainly focus on inhibiting the pathological NV with antiangiogenic compounds such as VEGF inhibitors and angiostatic steroids. In addition, laser photocoagulation of hypoxic retinal tissue with the idea of causing the regression of pathological NV, as the ischemic drive is decreased, is also being used. Promoting physiological revascularization by the modulation of MIP- $1 \beta$ in the hypoxic avascular retina during the early phase of ischemic retinopathy might be a new therapeutic method to prevent the vessel loss and retinal hypoxia, thereby suppressing subsequent pathological $\mathrm{NV}$ in hypoxia-induced vision-threatening retinal diseases.

\section{ACKNOWLEDGEMENTS}

We thank Ms Mari Imamura and Aiko Kuni (Kyushu University) for their excellent technical assistance. This work was supported in part by grants from the Ministry of Education, Science, Sports and Culture, Japan (TI and SY).

\section{DISCLOSURE/CONFLICT OF INTEREST}

The authors declare no conflict of interest. 
1. Ishikawa K, Yoshida S, Kadota K, et al. Gene expression profile of hyperoxic and hypoxic retinas in a mouse model of oxygen-induced retinopathy. Invest Ophthalmol Vis Sci 2010;51:4307-4319.

2. Schall TJ, Bacon KB. Chemokines, leukocyte trafficking, and inflammation. Curr Opin Immunol 1994;6:865-873.

3. Wolpe SD, Davatelis G, Sherry B, et al. Macrophages secrete a nove heparin-binding protein with inflammatory and neutrophil chemokinetic properties. J Exp Med 1988;167:570-581.

4. Koch $A E$, Kunkel SL, Shah MR, et al. Macrophage inflammatory protein1 beta: a C-C chemokine in osteoarthritis. Clin Immunol Immunopathol 1995;77:307-314.

5. O'Grady NP, Tropea M, Preas II HL, et al. Detection of macrophage inflammatory protein (MIP)-1alpha and MIP-1beta during experimental endotoxemia and human sepsis. J Infect Dis 1999;179:136-141.

6. Hasegawa M, Sato S, Takehara K. Augmented production of chemokines (monocyte chemotactic protein-1 (MCP-1), macrophage inflammatory protein-1alpha (MIP-1alpha) and MIP-1beta) in patients with systemic sclerosis: MCP-1 and MIP-1alpha may be involved in the development of pulmonary fibrosis. Clin Exp Immunol 1999;117:159-165.

7. Jo N, Wu GS, Rao NA. Upregulation of chemokine expression in the retinal vasculature in ischemia-reperfusion injury. Invest Ophthalmol Vis Sci 2003;44:4054-4060.

8. Ambati BK, Anand A, Joussen AM, et al. Sustained inhibition of corneal neovascularization by genetic ablation of CCR5. Invest Ophthalmol Vis Sci 2003;44:590-593.

9. Aiello LP, Avery RL, Arrigg PG, et al. Vascular endothelial growth factor in ocular fluid of patients with diabetic retinopathy and other retinal disorders. N Engl J Med 1994;331:1480-1487.

10. Gragoudas ES, Adamis AP, Cunningham Jr ET, et al. Pegaptanib for neovascular age-related macular degeneration. $N$ Engl J Med 2004;351:2805-2816.

11. Shimura $M$, Yasuda K. Macular ischaemia after intravitreal bevacizumab injection in patients with central retinal vein occlusion and a history of diabetes and vascular disease. Br J Ophthalmol 2010;94:381-383.

12. Saint-Geniez M, Maharaj AS, Walshe TE, et al. Endogenous VEGF is required for visual function: evidence for a survival role on muller cells and photoreceptors. PLoS One 2008;3:e3554.

13. Polverini PJ, Cotran PS, Gimbrone Jr MA, et al. Activated macrophages induce vascular proliferation. Nature 1977;269:804-806.

14. Ishida S, Usui T, Yamashiro K, et al. VEGF164-mediated inflammation is required for pathological, but not physiological, ischemia-induced retinal neovascularization. J Exp Med 2003;198:483-489.

15. Lima e Silva R, Shen J, Hackett SF, et al. The SDF-1/CXCR4 ligand/ receptor pair is an important contributor to several types of ocular neovascularization. FASEB J 2007;21:3219-3230.

16. Ritter MR, Banin E, Moreno SK, et al. Myeloid progenitors differentiate into microglia and promote vascular repair in a model of ischemic retinopathy. J Clin Invest 2006;116:3266-3276.

17. Smith LE, Wesolowski E, McLellan A, et al. Oxygen-induced retinopathy in the mouse. Invest Ophthalmol Vis Sci 1994:35:101-111.

18. Tachikawa T, Irie T. A new molecular biology approach in morphology: basic method and application of laser microdissection. Med Electron Microsc 2004;37:82-88.

19. Yoshida S, Yoshida A, Ishibashi T, et al. Role of MCP-1 and MIP-1alpha in retinal neovascularization during postischemic inflammation in a mouse model of retinal neovascularization. J Leukoc Biol 2003;73:137-144.

20. Yoshida S, Ogura A, Ishikawa K, et al. Gene expression profile of fibrovascular membranes from patients with proliferative diabetic retinopathy. Br J Ophthalmol 2009;94:795-801.
21. Connor KM, Krah NM, Dennison RJ, et al. Quantification of oxygeninduced retinopathy in the mouse: a model of vessel loss, vessel regrowth and pathological angiogenesis. Nat Protoc 2009;4: 1565-1573.

22. Pierce EA, Avery RL, Foley ED, et al. Vascular endothelial growth factor/ vascular permeability factor expression in a mouse model of retinal neovascularization. Proc Natl Acad Sci USA 1995;92:905-909.

23. Soulet $\mathrm{D}$, Rivest $\mathrm{S}$. Bone-marrow-derived microglia: myth or reality? Curr Opin Pharmacol 2008;8:508-518.

24. Sata M, Saiura A, Kunisato A, et al. Hematopoietic stem cells differentiate into vascular cells that participate in the pathogenesis of atherosclerosis. Nat Med 2002;8:403-409.

25. Rajantie I, Ilmonen M, Alminaite A, et al. Adult bone marrow-derived cells recruited during angiogenesis comprise precursors for periendothelial vascular mural cells. Blood 2004;104:2084-2086.

26. Mabuchi T, Kitagawa K, Ohtsuki T, et al. Contribution of microglia/ macrophages to expansion of infarction and response of oligodendrocytes after focal cerebral ischemia in rats. Stroke 2000;31:1735-1743.

27. Buttini M, Appel K, Sauter A, et al. Expression of tumor necrosis factor alpha after focal cerebral ischaemia in the rat. Neuroscience 1996;71: $1-16$.

28. Elkabes S, DiCicco-Bloom EM, Black IB. Brain microglia/macrophages express neurotrophins that selectively regulate microglial proliferation and function. J Neurosci 1996;16:2508-2521.

29. Lange C, Ehlken C, Stahl A, et al. Kinetics of retinal vaso-obliteration and neovascularisation in the oxygen-induced retinopathy (OIR) mouse model. Graefes Arch Clin Exp Ophthalmol 2009:247:1205-1211.

30. Ozaki $H, Y u A Y$, Della $N$, et al. Hypoxia inducible factor-1alpha is increased in ischemic retina: temporal and spatial correlation with VEGF expression. Invest Ophthalmol Vis Sci 1999;40:182-189.

31. Davies MH, Eubanks JP, Powers MR. Microglia and macrophages are increased in response to ischemia-induced retinopathy in the mouse retina. Mol Vis 2006;12:467-477.

32. Stahl A, Connor KM, Sapieha $\mathrm{P}$, et al. The mouse retina as an angiogenesis model. Invest Ophthalmol Vis Sci 2010;51:2813-2826.

33. Bosco MC, Reffo G, Puppo M, et al. Hypoxia inhibits the expression of the CCR5 chemokine receptor in macrophages. Cell Immunol 2004:228:1-7.

34. Uguccioni $M, D^{\prime} A p u z z o ~ M$, Loetscher $M$, et al. Actions of the chemotactic cytokines MCP-1, MCP-2, MCP-3, RANTES, MIP-1 alpha and MIP-1 beta on human monocytes. Eur J Immunol 1995; 25:64-68.

35. Davies MH, Stempel AJ, Powers MR. MCP-1 deficiency delays regression of pathologic retinal neovascularization in a model of ischemic retinopathy. Invest Ophthalmol Vis Sci 2008;49:4195-4202.

36. Bosco MC, Puppo M, Blengio F, et al. Monocytes and dendritic cells in a hypoxic environment: spotlights on chemotaxis and migration. Immunobiology 2008;213:733-749.

37. Zou H, Otani A, Oishi A, et al. Bone marrow-derived cells are differentially involved in pathological and physiological retinal angiogenesis in mice. Biochem Biophys Res Commun 2010;391: 1268-1273.

38. Penn JS, Tolman BL, Henry MM. Oxygen-induced retinopathy in the rat: relationship of retinal nonperfusion to subsequent neovascularization. Invest Ophthalmol Vis Sci 1994;35:3429-3435.

39. Aiello LP, Pierce EA, Foley ED, et al. Suppression of retinal neovascularization in vivo by inhibition of vascular endothelial growth factor (VEGF) using soluble VEGF-receptor chimeric proteins. Proc Natl Acad Sci USA 1995:92:10457-10461. 